\title{
BMJ Open Experiences of Australian primary healthcare nurses in using telehealth during COVID-19: a qualitative study
}

\author{
Sharon James (D) , ${ }^{1,2}$ Christine Ashley (i) , ${ }^{1}$ Anna Williams (D) , ${ }^{3}$ \\ Jane Desborough (D) , ${ }^{4}$ Susan Mcinnes (D) , Kaara Calma (i) , ${ }^{1}$ Ruth Mursa (i) , \\ Catherine Stephen (D) , ${ }^{1}$ Elizabeth J Halcomb (D) ${ }^{1}$
}

To cite: James S, Ashley C, Williams A, et al. Experiences of Australian primary healthcare nurses in using telehealth during COVID-19: a qualitative study. BMJ Open 2021;11:e049095. doi:10.1136/ bmjopen-2021-049095

- Prepublication history for this paper is available online. To view these files, please visit the journal online (http://dx.doi org/10.1136/bmjopen-2021049095).

Received 17 January 2021 Accepted 14 July 2021

\section{Check for updates}

(C) Author(s) (or their employer(s)) 2021. Re-use permitted under CC BY-NC. No commercial re-use. See rights and permissions. Published by BMJ.

${ }^{1}$ School of Nursing, Faculty of Science, Medicine and Health, University of Wollongong, Wollongong, New South Wales, Australia

${ }^{2}$ Department of General Practice, School of Public Health and Preventive Medicine, Faculty of Medicine, Nursing and Health Sciences, Monash University, Notting Hill, Victoria, Australia ${ }^{3}$ School of Nursing and Midwifery, Health Sciences and Physiotherapy, University of Notre Dame, Sydney, New South Wales, Australia

${ }^{4}$ Research School of Population Health, College of Health and Medicine, Australian National University, Canberra, Australian Capital Territory, Australia

Correspondence to Prof Elizabeth J Halcomb; ehalcomb@uow.edu.au

\section{ABSTRACT}

Objective This study sought to explore the experiences of Australian primary healthcare (PHC) nurses in the use of telehealth during COVID-19. Telehealth was defined as the use of any telecommunications mode (eg, telephone and videoconferencing) to deliver healthcare.

Design and setting Thematic analysis of qualitative interviews undertaken in Australian PHC.

Participants Twenty-five PHC nurses who had participated in a national survey about their experiences during COVID-19 were recruited using purposive sampling. Methods Semistructured telephone interviews were conducted from June to August 2020. Interviews lasted a mean of $38.5 \mathrm{~min}$. They were audio-recorded and transcribed before thematic analysis was undertaken. The consolidated criteria for reporting qualitative research were followed.

Results Four overarching themes were identified: preparedness, accessibility of telehealth, care experience and impacts on the PHC nurses' role. Some nurses were experienced in the use of telehealth, while others indicated a lack of preparation and limited appropriate technology to support its use. Telehealth enabled patients to access care but did not support complex clinical assessment. Participants indicated that patient engagement in telehealth was dependent on access and confidence using technology, perceived safety when physically attending the practice and the value they placed on care via telehealth. Many participants expressed frustration about telehealth funding and its impact on facilitating nurses to practise to their full scope.

Conclusion Telehealth has provided a means to continue PHC service delivery during COVID-19. While there are advantages to adopting this technology, considerations of the challenges and lessons from this experience are important to inform the future implementation of telehealth initiatives.

\section{INTRODUCTION}

The COVID-19 pandemic has prompted both tertiary care-based and community-based health services to rapidly assess their current service delivery models. Health services have been forced to implement ways of working that reduce the risk of viral transmission while at the same time providing quality
Strengths and limitations of this study

- Little is known about the role of primary healthcare (PHC) nurses using telehealth as a means to provide healthcare during COVID-19.

- This study explores nurses' experiences during COVID-19 which will help to inform models of telehealth into the future as part of both usual care and pandemic response.

- The study used qualitative interviews undertaken by nurses with PHC nursing research experience to support in-depth responses given by PHC nurse participants.

- There was diversity of participants in terms of employment setting, geographical location, experience and registration status.

- Purposive sampling of participants from a larger survey participant group was based on ensuring a representative mix of employment, $\mathrm{PHC}$ setting and registration status, as well as geographical location.

healthcare. ${ }^{12}$ The need for social distancing, shortages of personal protective equipment and a need to protect healthcare workers and patients prompted brisk exploration of service delivery options beyond face-to-face consultation. $^{2}$

Despite much of the attention early in the pandemic being focused on tertiary acute care services, primary healthcare (PHC) has an integral, front-line role in the pandemic response, including the management of people presenting with respiratory symptoms and/or fever, continued health management for those with chronic conditions, preventive activities such as vaccinations and community education around disease transmission. ${ }^{3}$ To ensure that these services were delivered, PHC services needed to pivot to new models of care.

While Australia has a system of universal health coverage funded through the national health insurance scheme, Medicare, the provision and funding of $\mathrm{PHC}$ is 
complex. PHC services are delivered by a combination of non-government organisations, state/territory-funded community health services and, small business and corporate chain general practices. ${ }^{4}$ This multitude of service providers and variations in the funding of services can impact the ability of the sector to effectively implement new models of service delivery.

Telehealth is an efficient, cost-effective and high-quality option to providing face-to-face health services ${ }^{2}$ involving health professionals using telecommunications and information technology to provide health assessment, diagnosis, intervention, consultation and health information to patients. Prepandemic telehealth research has demonstrated that telehealth has lower costs for both patients and healthcare professionals, with no change in service use or disease progression when compared with face-toface healthcare. ${ }^{56}$ Additionally, telehealth is associated with high levels of satisfaction among both patients and health professionals. ${ }^{56}$ However, despite the potential for telehealth to be a positive change in care delivery, organisational case studies have demonstrated that its introduction is a complex change. ${ }^{5}$ Concerns about the quality and safety of care, privacy and professional accountability have all been raised as issues associated with the widespread implementation of telehealth. Despite technological advancement offering the potential to provide healthcare via digital platforms, health regulation and funding structures in Australia have prevented widespread implementation of digital systems. ${ }^{27}$

In Australia, before the pandemic, funded telehealth was available only to people outside major cities and largely only for specialist consultations. ${ }^{8}$ In March 2020, the Australian government provided dedicated funding for Medicare-subsidised telehealth services (video or telephone) for all Australians who had an established relationship with a general practice. ${ }^{8}$ Implemented in eight stages from 13 March to 18 September 2020, these services initially prioritised vulnerable patients and providers, general practitioners (GPs), mental health providers and selected medical specialists. It was not until 20 April 2020 in stage 5 of telehealth implementation that funding was available to support general practice nurse (GPN) telehealth consultations, which were limited to health assessments for Aboriginal and Torres Strait Islander people, and chronic disease management consultations. ${ }^{9} 10$

The precipitous need for an alternative to face-to-face consultations has seen a rapid upscaling of telehealth in Australia and internationally. While this enabled health services to continue to provide care during the pandemic, ${ }^{11}$ it is important that lessons from this experience are used to inform ongoing care provision via telehealth and future pandemic response.

\section{METHODS}

This paper reports on the qualitative descriptive component of a mixed-methods study examining the experiences of Australian PHC nurses during the COVID-19 pandemic. Data in this paper specifically focused on nurses' experiences with telehealth. Given the volume and depth of data, other discrete aspects of their experience are reported separately. ${ }^{12-14}$

\section{Recruitment and sampling}

Australian PHC nurses who participated in a national survey about their experiences during COVID-19 ${ }^{13} 14$ were invited to participate in a qualitative semi-structured interview. Recruitment for the survey was undertaken through social media platforms and promoted through nursing professional organisations. Survey respondents indicating a willingness to be interviewed were purposively selected to provide a representation of employment setting (community-based and general practice), gender, location and registration status (enrolled nurse (EN), registered nurse (RN) and nurse practitioner (NP)). Prospective participants were approached to participate via phone and email. This contact provided study information, allowed participants to answer questions and sought written consent to participate.

\section{Data collection}

Individual telephone interviews were conducted between June and August 2020 by two female nurse researchers with experience in qualitative research and PHC nursing. Telephone interviews were used due to the geographical distribution of participants and pandemic restrictions. An interview schedule was developed from the survey findings and literature (box 1). Questions and prompts were designed by the research team to explore the nurses' perceptions and experiences about telehealth during COVID-19 based on the data from the initial survey. These questions were reviewed after six pilot interviews to ensure clarity. As no major changes were made, the pilot interviews were included in the dataset. All interviews were audio-recorded and transcribed verbatim by professional transcriptionists. Field notes were written to capture interviewer perceptions during data collection. Length of interviews ranged from $19.0 \mathrm{~min}$ to $59.0 \mathrm{~min}$ (mean $38.5 \mathrm{~min}, \mathrm{SD} 11.4 \mathrm{~min}$ ).

\section{Patient and public involvement}

As the research team included PHC nurses and feedback from nurses was available via the survey data, we did not engage nurses in the development of the interview questions. Nurses were involved in the recruitment to the initial survey as social media and snowballing methods

\section{Box 1 Excerpt from interview schedule}

How were you involved in telehealth in your practice?

To what extent was telehealth used to replace face-to-face care?

- How prepared were you for the introduction of telehealth?

What has helped/hindered the introduction of telehealth?

- How have your patients responded to care provided by telehealth?

- To what extent do you feel telehealth is effective in meeting the needs of patients? What do you think influences this? 
were used to recruit survey participants. Findings from the research are being disseminated back to nurses via social media and presentations to professional groups. Given the nature of the study, no patients were involved.

\section{Data analysis}

Transcripts were imported into Microsoft Word (Office 365) for analysis using the process described by Braun and Clark. ${ }^{15}$ Two researchers (CA and SJ) deeply immersed themselves in the data through reading and rereading transcripts and listening to the audio recordings. They then separately undertook manual coding of the transcripts to note interesting features. These interpretations were then cross-checked by AW and discussed with the broader team, focusing on the collation and verification of the codes and the development of emerging patterns and themes. Following this, each theme was defined and named and the transcripts were reviewed to ensure that all data were captured. The Consolidated Criteria for Reporting Qualitative Research checklist was used to guide the reporting. ${ }^{16}$

\section{RESULTS}

\section{Participants}

Twenty-five participants were interviewed (12 communitybased nurses (CNs) and 13 GPNs). The $12 \mathrm{CNs}$ worked in diverse clinical settings such as women's health, mental health, maternal and child health, and an Aboriginal medical service. These positions were funded through state/territory government funding or via nongovernment organisations. In contrast, the 13 GPNs were employed in general practices which were operated by either small businesses or corporate chains. Therefore, their employment was more reliant on income generated from service delivery and Australian government funding. Twenty-four (96\%) participants were female; 20 $(80 \%)$ were RNs; and 12 (48\%) had over 11 years' experience as PHC nurses.

Four overarching themes were identified that described participants' experiences of using telehealth during the COVID-19 pandemic, namely: preparedness, accessibility of care, care experience and impacts on the PHC nurses' role.

\section{Preparedness}

There was a diversity of preparedness among participants for the rapid implementation of telehealth. Preparedness was conceptualised in terms of both the available equipment and staff capacity in providing a telehealth service. Those who already used telehealth in their practice and had adequate resources found it easy to pivot to increasing telehealth use:

I think being rural helped us a lot 'cause we've done a lot of Skype stuff, ... - we'll set a person up in a room for a Skype consultation with a specialist ... that geographical isolation has actually made us much better at that sort of thing. (GPN10, RN)
... all the nurses have iPads. We all have phones, iPads....say, if they have a wound assessment, we've got a little camera and we could just take photographs of the wounds. (CN12, RN)

In contrast, others were less well prepared. The availability of equipment and the capacity of health professionals and patients to use audio-visual technologies resulted in most participants providing consultations via telephone.

A lot of people [health professionals] had never Zoomed or Skyped before, so everyone was sort of playing catch up. (CN01, RN)

We've got computers that don't have cameras and don't have microphones, so initially we didn't have the option. We've since changed that but we haven't had anyone interested in doing the conferencing. So we just do the phone. (GPN08, RN)

\section{Accessibility of care}

Most participants perceived telehealth to be a positive initiative that enhanced patients' access to care. This was balanced with concerns about engagement with telehealth due to confidence, the ability for nurses to effectively assess patients and access for vulnerable groups. For some, telehealth acceptability was seen through increased accessibility of services, particularly those who were living in rural areas.

we've got many patients that live in the bush. It's fantastic for them. Coming to town to see the doctor for a script, you know, it's a bit tough at the best of times. So they're loving it. (GPN05, EN)

Telehealth was also described as an acceptable way to maintain access to health services while avoiding home visits or high-risk patients attending clinics.

And I think for us it's useful in that it manages to reach patients that we wouldn't reach otherwise, because they wouldn't come in if they didn't have to because they're concerned about the risks. (GPN13, $\mathrm{RN})$

I had several oldies who are also having chemo, having radiotherapy, on immunosuppressants as well. They were running out of their medicines.... home isolating, really shutting themselves down from the world.... I did all telephone and it was so nice. I could hear their voice. I could tell by their speech, the tone in their voice, the conversation, how they were breathing during the conversation, .... and I was reassured that... they were fine, they were coping okay. (CN02, NP)

Other participants highlighted improved accessibility in terms of funding of telehealth services during the pandemic, which reduced the financial burden for patients. 
they're bulk-billed [no patient out of pocket expense]. That's a huge factor, I think. No one's got a bloody job. (GPN10, RN)

Enhanced accessibility was also highlighted by participants who described the time savings and efficiencies that could be achieved in simple consultations.

If you just need scripts or just like to get some routine blood tests done, where the appointment is gonna take five minutes in and out, telehealth is going to open up so many more appointments for the doctor. (GPN07, RN)

However, participants emphasised the importance of face-to-face consultations for more complex matters requiring physical assessment.

If it was the only means, it wouldn't be ideal, but it's fit for purpose. Some days, you'll look and say, 'Look, I can't do that over -" They'll show you something. I'll say, "I'll have to see that in real life. It's not clear enough there.' Or other days, it just fits perfectly. (GPN11, NP)

Participants also described how their experiences during COVID-19 have 'made us aware that ...we could look at a mix of - into the future, a mix of face-to-face and a mix of Telehealth, if we're able to do it' (CN09, RN).

Despite the enhanced accessibility of PHC services for many, the provision of equitable healthcare to vulnerable population groups such as the elderly, people with language barriers and those with chronic conditions, low income or homelessness were considered by some participants as challenging.

...mostly elderly [patients], they have mobile phones, but they wouldn't know how to use them.... They'd have a computer to do emails, but they wouldn't know how to do a Skype or anything like that. (CN02, NP)

...from a multicultural client background, we need a video conference platform where we can bring interpreters in... They can actually see the client, we can pick up all those nuances that are totally hidden if you're just on the phone. (CN10, RN)

\section{Care experience}

The extent of patient engagement in telehealth services depended on perceptions of vulnerability and safety during the pandemic, and preferences for face-to-face consultation, especially for elderly patients where the consultation ensured some social interaction. GPN01 (RN) identified that 'a lot of them [older people] were lonely too so, it was kind of a highlight for them sometimes to talk and just talk on the phone.'

Some participants perceived that patients felt that consultations provided via telehealth were below the usual standard of care. When consultations were undertaken via telephone 'some patients [were] not feeling like they're receiving a full service that they would be used to receiving' (GPN06, RN). GPN13 (RN) observed, 'I think for some patients, it was that they felt like because they were only hearing the voice, they couldn't read some of that nonverbal cue.... like if they could hear you talking and typing in the background, they weren't sure that you were actually listening to what they were saying.'

However, other participants found that their concern around patient preferences for face-to-face care was unfounded and that it was health professionals who were concerned about telehealth implementation.

So we've had it as part of our service plan... an increase in the use of videoconferencing, and we've always had a bit of resistance from clinicians... the consumers are fine. (CN08, RN)

... we are taught at uni to read a patient's body language, to triage them in terms of how they act and how they're talking, what they're wearing, and we're looking at their mental health in person... and you can't do that over the phone. So I think a lot of patient care was below our usual standard because we couldn't see our patients. (GPN04, RN)

\section{Impacts on the nursing role}

The rapid implementation of telehealth required participants to pivot their current role and workload to incorporate the new service delivery model. For those nurses working in general practice, the constraints of health system funding models were a significant impediment to their agility. However, community nurses described being able to redesign their service delivery models to incorporate telehealth. For example, they offered proactive services such as checking on patient welfare and exercise classes, as well as raising the profile of their service through social media.

I think that we were very proactive with our phone calls, so we phoned people... to make sure they are doing okay which we do proactively to prevent disasters happening... We really took an attitude that we wanted to keep people out of hospital. (CN08, RN)

we've done a lot of Zoom in women's health... we've done Zoom yoga. We've done a lot of sort of putting information onto our Facebook page. I think we've raised our profile because what we saw in the quarter with our stats was that we were getting a lot more new clients. (CN09, RN)

However, for GPNs, the funding of their employment meant that due to the initial restrictions of government funding of telehealth to doctors and allied health professionals, consultations were GP-led or had limited GPN involvement. GPNs described their frustration at these limitations, particularly in relation to providing COVID-19 education, triage, pathology or checking in on patient welfare or mental health.

the doctor doesn't need me to speak to the patients on the phone because they can't bill them, so that's not viable for the practice. (GPN08, RN) 
the doctors are doing all the phone calls and receiving the billings and we're not doing any care planning at all..... We're not even giving results 'cause it's not billable, whereas if the doctors give results, it's billable. (GPN10, RN)

Following the introduction of funding for GPN consultations in late April 2020, GPNs' involvement in telehealth was limited to activities attracting financial remuneration for the practice such as chronic disease management care plan preparation and nursing follow-up for those patients.

Well, I have, the care plans... We don't have any item numbers, so we can't bill anything. But helping the doctor set up the care plan and talking to the patients about their chronic disease prior to their appointment with the doctor, that's how we've done it. (GPN07, RN)

The delayed introduction of funding for GPN telehealth consultations impacted on nurses' job security.

But by the time they came in my work changes had already occurred. I think if maybe if they'd been a couple of weeks earlier there might have been a bit more ability for me to keep some work. But in my workplace, it didn't happen. (GPN01, RN)

Maintaining funding for GPNs to provide telehealth services was considered important to facilitate better use of their roles and to ensure that health assessments and other preventive or health promotion initiatives were maintained.

Look, I think access to Medicare, Telehealth for GPNs would be really important because I think sometimes - like people missing out on their over75 health checks, I think that in GP land, particularly I think nurses are underutilised in many respects. That's been my opinion for a long time. (CN09, NP)

\section{DISCUSSION}

\section{Summary}

This study has examined the experiences of Australian PHC nurses in the rapid deployment of telehealth during the COVID-19 pandemic. Participants identified challenges in preparedness in terms of the availability of equipment and staff skill levels. While for some nurses, telehealth enhanced their capacity to facilitate healthcare access for patients, for those without access to appropriate technology, access was reduced. Patient engagement in telehealth consultations and recognition of the value of healthcare delivered via telehealth were cited as a potential concern. Finally, participants described the impact of funding models for telehealth services on the nursing role, particularly in general practice, and highlighted the importance of funding to maintain job security and to enable GPNs to work to the extent of their scope of practice. These factors highlight the complexity of change required to effectively embed telehealth within the healthcare system and the need for organisational and health system support to address these issues at a practice level.

\section{Strengths and limitations}

The inclusion of nurses from across diverse PHC settings is a strength of this study as it provides broad perspectives of the experience of nurses across the community sector. The mostly female participant group reflects the femaledominated nature of the nursing profession, despite our attempts to recruit male nurses. While telephone interviews allowed us to overcome barriers around geographical dispersion and pandemic precautions, these may have impacted the quality of data collected compared with face-to-face interviews. Additionally, although the inclusion of data from patients and other members of the multidisciplinary team may have provided depth and richness, it was beyond the scope of this study.

\section{Comparison with existing literature}

Previous telehealth research has focused on organisational factors or patient and provider experiences in the planned implementation of telehealth programmes. ${ }^{17}$ The need for complex organisational change to support the effective implementation of telehealth has been identified. ${ }^{5}$ This current study reported the rapid upscaling of telehealth services to maintain safe primary healthcare in response to the COVID-19 pandemic. The COVID-19 pandemic transformed $\mathrm{PHC}$ overnight, requiring care to be provided at a distance while maintaining the ongoing relationships that characterise PHC. $^{18}$ The rapid and unexpected widespread deployment of telehealth during COVID-19, combined with the stresses on the healthcare system, challenged health professionals to effectively implement such complex change within very short time frames. The lessons learnt from this rapid deployment of telehealth likely have implications for both future pandemic response and the widespread implementation of telehealth beyond the pandemic.

Unlike other countries, in Australia, government funding was equivalent for both video and telephone consultations. However, in this study, participants largely described telephone consultations due to the availability of equipment and rapid upscaling. While many simple consultations can be effectively managed via telephone, video adds visual cues, ${ }^{19}$ and face-to-face contact is likely preferable for complex health issues. ${ }^{20}$ In contrast, at times, video consultations can compromise an individual's privacy, whereas telephone consultations may enable the patient to feel as if the contact is more private. ${ }^{21}$ Further research is likely needed around the relative effectiveness of various modes of telehealth for the range of health care services delivered in PHC.

This study found that telehealth increased access for many patients who had not previously had access to such services, as it overcame burdens around travel time and waiting for consultations. Telehealth has been increasingly used in rural and regional areas for this reason. ${ }^{22}$ 
Consistent with the literature, this study noted challenges to patient access related to vulnerabilities such as age, language and disability. ${ }^{23}$ Disparity in access to telehealth is often experienced for those at highest risk, with low health literacy and with the most limited access to technology ${ }^{21}$ Facilitation of access to telehealth within the community is essential to ensure equity for all.

Despite improvements in access to healthcare created by telehealth, this study raised issues around patients and health professional concerns about the impact of telehealth on the care experience. Concerns about adequate physical assessment, feeling as if they are receiving less attention, being reluctant to ask questions, feeling rushed and less involved in their care, and having a poorer relationship with their health professional have all been identified by patients as concerns about telehealth consultations. ${ }^{24}$ While face-to-face consultation options are not always possible during the pandemic, there is a need for nurses to actively seek to develop their practice to optimise the nurse-patient relationship within telehealth consultations. Strategies such as using telehealth methods that allow visual cues (eg, video), considering non-verbal expression, encouraging patients to engage in active communication, avoiding the perception of being rushed, and both critically reflecting on consultations and seeking feedback from patients can assist in optimising the quality of consultations and care experience. ${ }^{24}$ Given that it is unlikely that our health systems will return to prepandemic levels of telehealth use, nursing education programmes must consider the skills and education related to providing nursing care via telehealth that is needed by nurses to work effectively in our future health systems.

Given the reliance of Australian general practices on the Medicare Benefits Schedule and funding incentives to employ nurses, this study found that the initial exclusion of nurses from changes to the funding to support telehealth was problematic. During the period from March to April 2020, when telehealth was funded for GPs and allied health but not for GPNs ${ }^{25}$ the role of the nurse was significantly constrained. A similar story was reported by Watkins and Neubrander, ${ }^{26}$ who describe how American nurses were prohibited from providing reimbursable telehealth services. The impact of funding models limiting GPN roles in Australia is not new ${ }^{27}$; however, the impact seen during COVID-19 highlights the need for funding to support rather than inhibit health professionals to work to the full extent of their scope of practice to optimise service delivery during crises. ${ }^{1314}$

\section{Implications for practice}

The disruptive impact of COVID-19 has rapidly progressed the implementation and use of telehealth in Australian PHC as has occurred internationally. The results of this study indicate that while PHC nurses have embraced this implementation and highlighted the advantages of telehealth in promoting access to care during the pandemic, several factors need to be addressed if telehealth is embedded in the new normal. This study has highlighted that health professionals need ongoing education in both the use of technology and the delivery of telehealth consultations to optimise the care that they provide. Additionally, support needs to be given to patients to adapt to and access telehealth services. Finally, this study recognised the value in embracing this opportunity to embed the advancements in telehealth into models of usual care. However, telehealth will only be a viable mode of healthcare delivery if it is supported by appropriate funding mechanisms to support the provision of quality technology and the remuneration of multidisciplinary health professionals.

\section{CONCLUSION}

The PHC nurses in this study showed ways in which care can be continued during a pandemic. For health professionals to provide care via telehealth, appropriate support is needed. This includes ensuring health professionals and patients are prepared and engaged in telehealth as a mode of care through confidence in its use and access to technology. Additionally, the use of telehealth requires consideration of the need for in-person assessment versus physical distancing. However, to provide this care, telehealth funding needs to address its impact on PHC nurses to practise to their full scope.

Twitter Sharon James @Sharon_MJames, Jane Desborough @Jane0Des, Susan Mcinnes @sue_mcinnes, Kaara Calma @KaaraCalma, Ruth Mursa @RuthMursa, Catherine Stephen @CatMStephen and Elizabeth J Halcomb @LizHalcomb

Acknowledgements We thank the PHC nurses who generously participated in this study. Thanks also go to the Australian College of Nursing and Australian Primary Health Care Nurses Association for supporting this work.

Contributors SJ, CA, AW, JD, SM, KC, RM, CS and EJH made substantial contributions to the conception and design of the study and the interpretation of the data. SJ and CS conducted the interviews. SJ, CS and AW performed the analysis. SJ, CA, AW, JD, SM, KC, RM, CS and EJH contributed to the drafting of the paper and revised the paper's clarity and content before approving the paper for publication.

Funding This Project was jointly funded by the Australian College of Nursing and the University of Wollongong (award/grant number is not applicable).

\section{Competing interests None declared.}

Patient and public involvement Patients and the public were involved in the design, conduct, reporting or dissemination plans of this research. Refer to the Methods section for further details.

Patient consent for publication Not required.

Ethics approval The study was approved by the Human Research Ethics Committee of the University of Wollongong (approval number HE2020/161) and ratified by the University of Notre Dame Australia (approval number 2020-056S).

Provenance and peer review Not commissioned; externally peer reviewed.

Data availability statement № data are available. № data are publicly available.

Open access This is an open access article distributed in accordance with the Creative Commons Attribution Non Commercial (CC BY-NC 4.0) license, which permits others to distribute, remix, adapt, build upon this work non-commercially, and license their derivative works on different terms, provided the original work is properly cited, appropriate credit is given, any changes made indicated, and the use is non-commercial. See: http://creativecommons.org/licenses/by-nc/4.0/.

ORCID iDs

Sharon James http://orcid.org/0000-0003-2211-3447 
Christine Ashley http://orcid.org/0000-0003-0559-9553

Anna Williams http://orcid.org/0000-0003-0349-4248

Jane Desborough http://orcid.org/0000-0003-1406-4593

Susan Mcinnes http://orcid.org/0000-0003-3113-2930

Kaara Calma http://orcid.org/0000-0001-9011-368X

Ruth Mursa http://orcid.org/0000-0002-5329-7181

Catherine Stephen http://orcid.org/0000-0002-3864-1300

Elizabeth J Halcomb http://orcid.org/0000-0001-8099-986X

\section{REFERENCES}

1 Peine A, Paffenholz P, Martin L, et al. Telemedicine in Germany during the COVID-19 pandemic: Multi-Professional national survey. $J$ Med Internet Res 2020;22:e19745.

2 Kichloo A, Albosta M, Dettloff K, et al. Telemedicine, the current COVID-19 pandemic and the future: a narrative review and perspectives moving forward in the USA. Fam Med Community Health 2020;8.

3 Peate I. What nurses do as routine is special in the time of COVID-19. Practice Nursing 2020;31:308-9.

4 Duckett S, Willcox S. The Australian health care system. Oxford University Press, 2015.

5 Greenhalgh T, Wherton J, Shaw S, et al. Video consultations for COVID-19. BMJ 2020;368:m998.

6 Day K, Hunter I, Rao V, et al. Survey protocol for exploring video and phone use in Aotearoa New Zealand general practice: considerations for future telehealth. BMJ Health Care Inform 2021;28:e100309.

7 Wood SM, White K, Peebles R, et al. Outcomes of a rapid adolescent telehealth scale-up during the COVID-19 pandemic. J Adolesc Health 2020;67:172-8.

8 Snoswell CL, Caffery LJ, Haydon HM, et al. Telehealth uptake in general practice as a result of the coronavirus (COVID-19) pandemic. Aust Health Rev 2020;44:737.

9 Australian Government Department of Health. COVID-19 temporary MBS telehealth services 2020. Available: http://www.mbsonline.gov. au/internet/mbsonline/publishing.nsf/Content/0C514FB8C9FBBEC7 CA25852E00223AFE/\$File/COVID-19\%20Bulk-billed\%20MBS\% 20telehealth\%20Services\%20-\%20Overarching\%20200420.pdf [Accessed 29 Apr 2020].

10 Australian Government Department of Health. COVID-19 telehealth items guide, December 8, 2020, 2020. Available: https://www.health. gov.au/sites/default/files/documents/2020/12/coronavirus-covid-19telehealth-items-guide.pdf [Accessed 21 Dec 2020].

11 Desborough J, Hall Dykgraaf S, de Toca L, et al. Australia's national COVID-19 primary care response. Med J Aust 2020;213:104-6.

12 Ashley C, James S, Stephen C. Primary health care nurses' perceptions of risk during COVID-19: a qualitative study. J Nurs Scholar.
13 Halcomb E, Mclnnes S, Williams A, et al. The experiences of primary healthcare nurses during the COVID-19 pandemic in Australia. J Nurs Scholarsh 2020;52:553-63.

14 Halcomb E, Williams A, Ashley C, et al. The support needs of Australian primary health care nurses during the COVID-19 pandemic. J Nurs Manag 2020;28:1553-60.

15 Braun V, Clarke V. Using thematic analysis in psychology. Qual Res Psychol 2006;3:77-101.

16 Tong A, Sainsbury P, Craig J. Consolidated criteria for reporting qualitative research (COREQ): a 32-item checklist for interviews and focus groups. Int J Qual Health Care 2007;19:349-57.

17 MacNeill V, Sanders C, Fitzpatrick R, et al. Experiences of front-line health professionals in the delivery of telehealth: a qualitative study. Br J Gen Pract 2014;64:e401-7.

18 Westfall J, Stange K, DeVoe J. Coronavirus: family physicians provide telehealth care at risk of bankrutcy. USA Today, 2020. Available: https://www.usatoday.com/story/opinion/2020/04/07/coronavirusfamily-physicians-provide-telehealth-care-risk-bankruptcy-column/ 2942535001/

19 Greenhalgh T, Koh GCH, Car J. COVID-19: a remote assessment in primary care. BMJ 2020;368:m1182.

20 Blumenthal $D$. Where telemedicine falls short: the Commonwealth fund, 2020. Available: https://www.commonwealthfund.org/blog/ 2020/where-telemedicine-falls-short [Accessed 14 Jul 2020].

21 Health Information Technology Evaluation and Quality Center. Telehealth considerations and strategies for special and vulnerable populations, 2020. Available: https://hiteqcenter.apps.plantanapp. com/Resources/HITEQ-Resources/telehealth-considerations-andstrategies-for-special-and-vulnerable-populations [Accessed $3 \mathrm{Dec}$ 2020].

22 Jetty A, Moore MA, Coffman M, et al. Rural family physicians are twice as likely to use telehealth as urban family physicians. Telemed J E Health 2018;24:268-76.

23 Koivunen M, Saranto K. Nursing professionals' experiences of the facilitators and barriers to the use of telehealth applications: a systematic review of qualitative studies. Scand J Caring Sci 2018;32:24-44.

24 Gordon HS, Solanki P, Bokhour BG, et al. "I'm not feeling like I'm part of the conversation" patients' perspectives on communicating in clinical video telehealth visits. J Gen Intern Med 2020;35:1751-8.

25 Australian Government Department of Health. Medicare benefits schedule, temporary COVID-19 MBS telehealth services - Factsheet, September 18, 2020. Available: http://www.mbsonline.gov.au/ internet/mbsonline/publishing.nsf/Content/0C514FB8C9FBBEC7 CA25852E00223AFE/\$File/Factsheet-COVID-19-Bulk-billed-MBS\% 20telehealth-Services-Overarching-17.09.2020.pdf [Accessed 21 Dec 2020].

26 Watkins S, Neubrander J. Primary-care registered nurse telehealth policy implications. J Telemed Telecare 2020;1357633X:2094014.

27 Halcomb EJ, Davidson PM, Salamonson Y, et al. Nurses in Australian general practice: implications for chronic disease management. $J$ Clin Nurs 2008;17:6-15. 